\title{
Sepsis: implication for nursing
}

\author{
Fabio Bertoncini, ${ }^{1}$ Dino Stefano Di Massimo, ${ }^{1}$ Claudia Gatta ${ }^{2}$ \\ ${ }^{1}$ Division of Nursing, Department of Internal Medicine, Biella Hospital; ${ }^{2}$ Division of Nursing, Department of Medicine and \\ Emergency, Biella Hospital, Biella, Italy
}

\begin{abstract}
Sepsis is a serious health problem that is off worldwide interest; it is associated with a high mortality rate despite continuing improvements in infection management and the awareness of population is still poor despite its importance. Direct interventions to achieve the goal of clinician, like reduction of mortality, pass through the resuscitation and antibiotics but their effectiveness depends on the early recognition of symptoms and therefore the septic state. The nursing role is crucial both for the early recognition of the disease state, as well as to treat the patient with professionalism and promptness and to provide appropriate assistance to the kind of complexity that creates this pathological state: to achieve these aims, recommendations and bundles were developed to guide clinical nurses in septic patients' care.
\end{abstract}

\section{Introduction}

Sepsis is a serious health problem that is off worldwide interest; it is associated with a high mortality rate despite continuing improvements in infection management $^{1,2}$ and the awareness of population is still poor despite its importance. ${ }^{3}$

Rate of worldwide incidence is varied, but is estimated to occur in about 300 cases $/ 100,000$ people every year and the rate of mortality is comparable to the rate of mortality recorded in 1960 for myocardial infarction. The Agency for Healthcare Research and Quality considers sepsis the most expensive condition treated in US hospitals, in fact this condition costed in 2011 more than 20 billion dollars and about 2.5 billion pounds in the UK. ${ }^{3,4}$

Nurses approach septic patients every day and in all

Correspondence: Fabio Bertoncini, Division of Nursing, Department of Internal Medicine, Biella Hospital, via dei Ponderanesi 2, 13875 Ponderano (BI), Italy.

Tel.: +39.015.15153242.

E-mail: fabio.bertoncini@hotmail.it

Key words: Sepsis; nursing; bundle; clinical competence; medical patient.

Received for publication: 6 October 2016.

Revision received: 27 October 2016.

Accepted for publication: 27 October 2016.

This work is licensed under a Creative Commons Attribution NonCommercial 4.0 License (CC BY-NC 4.0).

CCopyright F. Bertoncini et al., 2016

Licensee PAGEPress, Italy

Italian Journal of Medicine 2016; 10:360-363

doi:10.4081/itjm.2016.801 areas, from community to hospital for acute patients. ${ }^{5}$

Direct interventions to achieve the goal of clinician, like reduction of mortality, pass through the resuscitation and antibiotics but their effectiveness depends on the early recognition of symptoms and therefore the septic state; for this reason the nursing role is crucial both for the early recognition of the disease state, as well as to treat the patient with professionalism and promptness ${ }^{6}$ and to provide appropriate assistance to the kind of complexity that creates this pathological state.

\section{Definition of sepsis}

The term sepsis was widely used in nursing practice in the past but this word has been misused because of unclear understanding of the meaning, so in 2012 it was defined by the Surviving Sepsis Campaign as: the presence (probable or documented) of infection together with systemic manifestations of infection. ${ }^{7}$

The body's response to sepsis is complex: by stimulating the immune system, white blood cells and responses of endothelial cells release cytokines and mediators in turn are activated. These in turn activate a series of physiological changes that include vasodilatation, increased capillary permeability, thrombophilia and decreased fibrinolytic processes. Although the immune response is a protective effect for the organism, the great production of cytokines and mediators damages the endothelial cells, capillary permeability changes and occurs an important vasodilation with consequent hypotension (severe sepsis).

All these immune responses that occur during the septic state, can lead to multiple organ failure. ${ }^{1,5}$ This can be considered a continuum that can evolve from one infection status to multi-organ failure that could lead to death. ${ }^{8}$ 
Sepsis is a serious medical condition that can develop in neonatal, pediatric and adult patients.

It can be caused by an infection that may occur in any part of the body and this infection may be caused by different microorganisms, which in $90 \%$ of cases are bacteria, but can also be caused by fungal infections or viruses. ${ }^{5,9-11}$

Bacteria associated with sepsis shock may be Gram-negative type [Escherichia coli, Klebsiella spp., Enterobacter spp., Pseudomonas aeruginosa, Serratia, Proteus, Bacteroides fragilis (anaerobe)] and Gram-positive type (Staphylococcus aureus, Pneumococcus, Alpha- and beta-hemolytic streptococci). ${ }^{12}$

The origin of sepsis is varied, it was found that sepsis of respiratory origin is the most common in the industrialized world ${ }^{4,5,13-15}$ with an incidence rate ranging between $35 \%$ and $50 \%$, followed by infections of urinary tract, intra-abdominal, catheter-related blood stream infection, device-related, central nervous system and others (e.g., cellulitis, intra-articular). ${ }^{4}$

\section{Nursing management of septic patient}

It is very difficult to identify, especially at early stage, sepsis because of the variability of the symptoms; for example, in $60 \%$ of patients with sepsis, the pathological state could present without fever, or in older people, a fall could hide a latent sepsis. ${ }^{4}$

For this reason, the Surviving Sepsis Campaign, in 2012 drew up international guidelines for the identification and management of severe sepsis. Generally, main signs of sepsis are: $\left.:^{2,7} \mathrm{i}\right)$ fever $\left(\mathrm{T}^{\circ}>38.3^{\circ}\right)$; ii) hypothermia $\left(\mathrm{T}^{\circ}<36^{\circ}\right)$; iii) hearth rate $>90 / \mathrm{min}$ (considers patient age); iv) tachypnea; v) altered mental status; vi) significant edema or positive fluid balance; vii) hyperglycemia (plasma glucose $>140$ $\mathrm{mg} / \mathrm{dL}$ ) in the absence of diabetes mellitus; viii) leukocytosis [white blood cells (WBC) count $>12,000$ cells $/ \mathrm{mm}^{3}$ ]; ix) leukopenia (WBC count $<4000$ cells $/ \mathrm{mm}^{3}$ ); x) low blood pressure (systolic $<90 \mathrm{mmHg}$, diastolic blood pressure $<70 \mathrm{mmHg}$, data must be associated with the patient's age). ${ }^{7}$

The above-mentioned guidelines have been drafted to give indications to the medical management of the patient in septic state but do not treat the importance of the nursing role in achieving a good outcome for the patient. Expert nurses with advanced skills are needed to identify patients with deterioration of clinical status and to ensure appropriate assistance to patients with sepsis. ${ }^{2}$

A study performed in $2011,{ }^{2}$ identified sixty-three recommendations to guide clinical nurses who care for patients with sepsis. The macro subjects are:

- Prevention of infection: the subject is education of staff which happens to be the first step (fundamental) to raise awareness of the problem and implement strategies for preventing infection, the accountability which results in the promotion of a culture of patient safety, the continuous surveillance of nosocomial infections, washing hands as evidence suggests that a proper hand washing reduces the rate of infections or alternatively the use of hydro-alcoholic gel and the proper use of those devices that can be vehicles of infection (e.g., mechanical ventilation patients, venous catheter-related infections, surgical-site infections and urinary tract infections).

- Management of infections: the topic is concerned with the identification of the source of infection and the early removal of the infected device or what purports to be is a critical step and prevention of transmission of infection among patients, particularly for infections of alert microorganism like methicillinresistant $S$. aureus or as Clostridium difficile.

- The first therapeutic approach: the nurse is responsible for the early recognition of septic state of the patient, clinical monitoring, collection of blood samples and not, administration of the antibiotic therapy, infusion therapy; the role of the nurse is fundamental throughout the patient care process in an area of great collaboration with various professional roles.

- Other nursing support: providing nutritional therapy in critically ill patients because malnutrition is associated with increased morbidity and mortality so that the guidelines suggest starting enteral nutrition in the first $24 / 48 \mathrm{~h}$, the eye care through hydration with ophthalmologic product, especially in patients in intensive care unit with altered levels of consciousness and who cannot hydrate their corneas and to protect them from dehydration, abrasion, perforation and infection, prevention of pressure ulcers through the mobilization and the use of anti-bedsore devices. ${ }^{1,2}$

For management of sepsis in acute moment, the fundamental approach includes early recognition, appropriate, and timely delivery of antibiotics, controlling the source of infection and adequate resuscitation with intravenous fluids and possibly vasoactive drugs. ${ }^{16}$

In 2014, a project named The Sepsis Six ${ }^{17}$ was successfully launched to improve management of septic patient. The sepsis six is comprised of three diagnostic and monitoring steps and three therapeutic interventions: i) deliver high-flow oxygen; ii) take blood cultures prior to antibiotics but do not delay treatment; iii) administer empirical intravenous antibiotics; iv) measure serum lactate; v) start intravenous fluid resuscitation with crystalloids; vi) commence urine output monitoring via either a catheter or chart.

Generally, sepsis bundles are a great approach to standardize care, and in some cases, to prevent the onset of the sepsis avalanche regretfully, they are no treatment for sepsis. ${ }^{8}$

The bundle implementation should consider: i) performing baseline observation of vital signs: respiration 
rate, oxygen saturation $\left(\mathrm{SpO}_{2}\right)$, capillary refill time, heart rate, and blood pressure. Consider the patient's level of consciousness - is he or she alert or drowsy? Level of consciousness is represented by the $\mathrm{D}$ - disability of the $\mathrm{ABCD}$ approach and refers to disability of the central nervous system; ${ }^{5}$ ii) making available the necessary for admistered supplemental oxygen therapy via a facial mask and necessary for obtaining blood specimens especially blood culture: ${ }^{5}$ blood cultures should be taken before antibiotics are given to identify any micro-organisms that may be in the blood. They should be taken from a peripheral vein and from any invasive catheters that the patient may have in situ, such as a central venous catheter. Blood cultures, however, are not always positive in patients with severe sepsis: ${ }^{18}$ iii) inserting a urinary catheter with an hour urometer that allows the hourly urine volume to be measured and to obtain a urine specimen; ${ }^{5}$ iv) considering pain and discomfort: pain and discomfort are the uppermost stressors for the critically ill, and apparent tissue damage is not a prerequisite for pain. Endotracheal suctioning (if necessary) hurts, prolonged immobilization and routine care are painful, and general intense discomfort of vague origin is common in critical illness; ${ }^{19,20}$ pain is one of major issue in sepsis and requires a continuous monitoring $;{ }^{8} \mathrm{v}$ ) observing the patient: to perform halfhourly observations of vital signs and hourly measurement of urinary output, ${ }^{5,7} \mathrm{vi}$ ) considering patient and family comfort/care: to promote patient comfort/pain relief/sedation/turning/skin care; provide patient and family teaching; address needs of families of critically ill patients. ${ }^{2}$

We can also consider these recommendations like the interventions for the main nursing diagnoses identified in patients with sepsis in 2014:21 the main nursing diagnoses, described according to the NANDA-I taxonomy are: risk for infection, risk for aspiration, risk for impaired skin integrity, impaired skin integrity, impaired spontaneous ventilation, impaired gas exchange.

A fundamental aspect is the condivision of nurses care goal with patients and their family. The literature may want to discuss the prognosis and goals of care such as ask no more than $72 \mathrm{~h}$ after admission to intensive care and then by the fact acute. The recommendations emphasize that it is essential to share the clinical situation with the patient and relatives, sharing the therapeutic objectives, flexible hours for the visit of the relatives and the integration of palliative support where necessary. Sometimes, the family of the patient must make a big effort to understand the criticality of the clinical picture and the nurse plays a fundamental role which is recognized by the international literature, to help family members to understand the situation, through continuous reports and talks. ${ }^{1,22,23}$

Generally, for treatment of hospitalized patients with sepsis, it is fundamental: ${ }^{6}$ i) to disseminate infor- mation on the new guidelines to the team members; ii) to include discussion of the guidelines during unit clinical care meetings and clinical rounds; iii) to constitute a multidisciplinary team and outline a timeline for implementing the guidelines; iv) to use the new guidelines as a performance improvement initiative for the Intensive Care Unit as well as noncritical care areas to improve recognition and treatment of patients with sepsis.

Specific areas include: i) aid in the early identification of sepsis including recognizing patients at risk for developing sepsis (patients who are elderly, immunocompromised, patients with surgical/invasive procedures, indwelling catheters, mechanically ventilated patients) and monitoring physical assessment parameters including vital signs and perfusion status (urine output, mental status changes, skin color); ii) to provide comprehensive sepsis treatment (circulatory support with fluids, inotropes, and vasopressors; supportive treatment with oxygenation and ventilation; antibiotic administration; sepsis guideline recommendation measures; monitor and report patient response to treatment); iii) to promote patient and family centered care (patient and family teaching, addressing the needs of families of critically ill patients, setting goals of care, and holding family care conferences to discuss goals of care).

It can be said then that nurses have a very specific and crucial role throughout the diagnostic and therapeutic process of the patient with sepsis. Guidelines and recommendations in the literature can help the nursing staff in developing information and to provide nursing care based on solid evidence to help achieving optimal patient outcomes.

\section{References}

1. Kleinpell R, Aitken L, Schorr CA. Implications of the new international sepsis guidelines for nursing care. Am J Crit Care 2013;22:212-22.

2. Aitken LM, Williams G, Harvey M, et al. Nursing considerations to complement the Surviving Sepsis Campaign guidelines. Crit Care Med 2011;39:1800-18.

3. Singer M, Deutschman C, Seymour C, et al. The Third International Consensus Definitions for Sepsis and Septic Shock (Sepsis-3). JAMA 2016;315:801-10.

4. An Roinn Slainte, Irish Department of Health. Sepsis Management: National Clinical Guideline No. 6; 2015. Available from: http://health.gov.ie/blog/publications/sepsis-management-national-clinical-guideline-no-6/

5. Robson W, Newell J. Assessing, treating and managing patients with sepsis. Nurs Stand 2005;19:56-64.

6. Kleinpell R. Updates in sepsis management: new guidelines for sepsis care. World Crit Care Nurs 2012;9:33-4.

7. Dellinger RP, Levy MM, Rhodes A, et al. Surviving sepsis campaign: international guidelines for management of severe sepsis and septic shock: 2012. Crit Care Med 2013;41:580-637.

8. Dunkley S, McLeod A. Neutropenic sepsis: assessment, 
pathophysiology and nursing care. Br J Neurosci Nurs 2015;11:79-87.

9. Angus DC, van der Poll T. Severe sepsis and septic shock. N Engl J Med 2013;369:840-51.

10. Cohen J. The immunopathogenesis of sepsis. Nature 2002;420:885-91.

11. Kim WS, Lee HJ. Management of sepsis. J Korean Med Assoc 2013;56:819-26.

12. Edwards S. Shock: types, classifications and explorations of their physiological effects. Emerg Nurse 2001;9:29-38.

13. Mayr FB, Yende S, Angus DC. Epidemiology of severe sepsis. Virulence 2014;5:4-11.

14. Sagy M, Al-Qaqaa Y, Kim P. Definitions and pathophysiology of sepsis. Curr Probl Pediatr Adolesc Health Care 2013;43:260-3.

15. Wheeler AP, Bernard GR. Treating patients with severe sepsis. N Engl J Med 1999;340:207-14.

16. ARISE Investigators; ANZICS Clinical Trials Group, Peake SL, et al. Goal-directed resuscitation for patients with early septic shock. N Engl J Med 2015;371: 1496-506.
17. McGregor C. Improving time to antibiotics and implementing the "Sepsis 6." BMJ Qual Improv Reports 2014;2:pii:u202548.w1443.

18. Alahmadi YM, Aldeyab MA, McElnay JC, et al. Clinical and economic impact of contaminated blood cultures within the hospital setting. J Hosp Infect. 2011;77:233-6.

19. Cooke SE, Dasta J, Fish D, et al. Clinical practice guidelines for the sustained use of sedatives and analgesics in the critically ill adult. Am J Health-Syst Pharm 2002;59: 150-78.

20. Elliott D. Discomfort and factual recollection in intensive care unit patients. Austr Crit Care 2005;18:43-4.

21. Dutra C, Silveira L, Santos A, Pereira R, Stabile A. Prevalent nursing diagnosis in patients hospitalized with sepsis at the intensive care unit. Cogit Enferm 2014;19:688-94.

22. Hanks RG. Social advocacy: a call for nursing action. Pastoral Psychol 2013;62:163-73.

23. Lindahl B, Sandman PO. The role of advocacy in critical care nursing: a caring response to another. Intensive Crit Care Nurs 1998;14:179-86. 